\title{
Hacia una concepción experimental de la filosofía en la escuela
}

\section{Towards an experimental conception of philosophy in school}

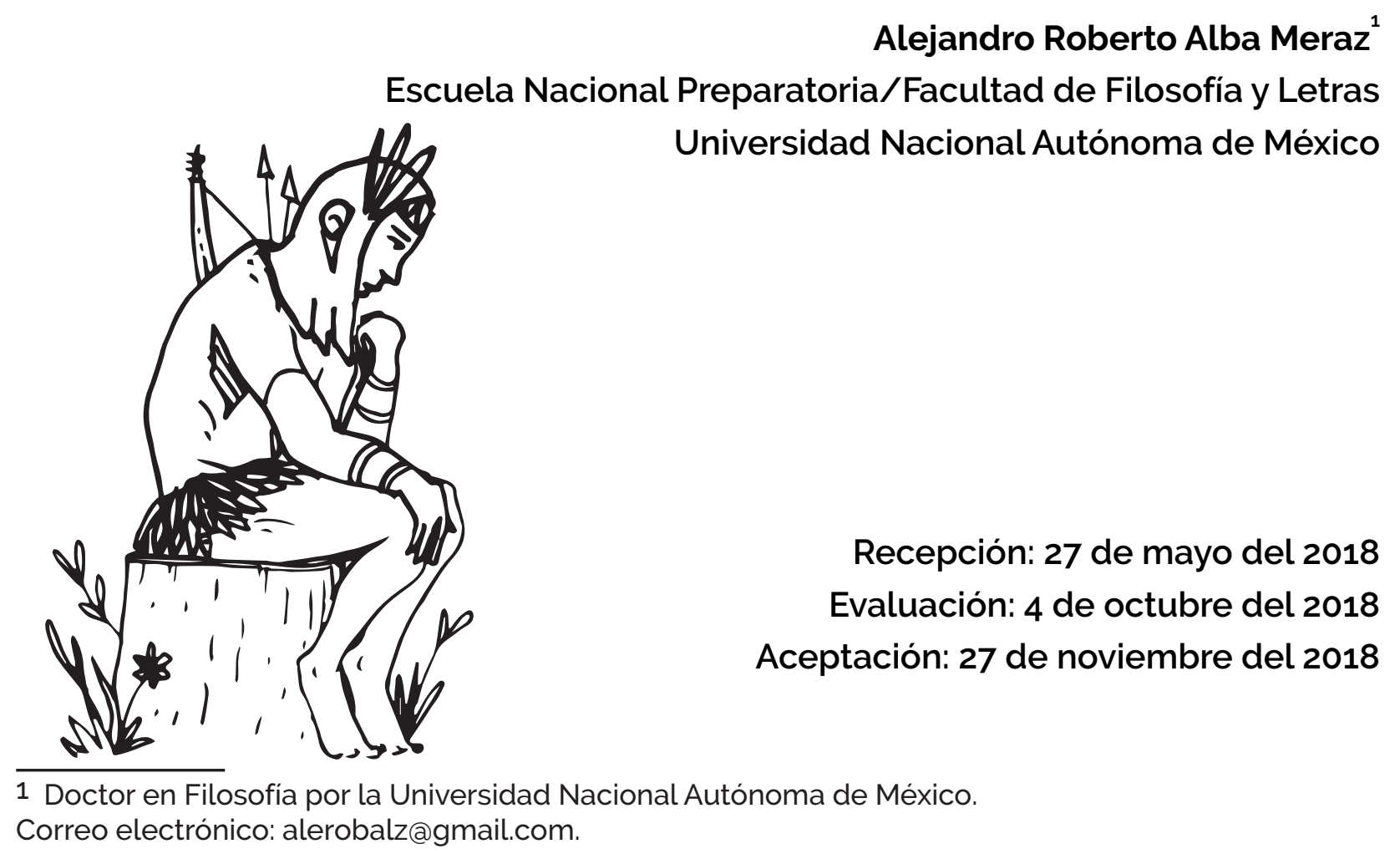




\section{Resumen:}

El artículo discute el papel que ocupa la filosofía dentro del currículum, a la luz de los cambios experimentados por el sistema educativo en las últimas décadas. En la actualidad, se reconoce que la escuela ha modificado su foco de atención y perspectiva, pues ha pasado de ser proveedora de conocimiento y guía de una formación humanística a gestora de competencias y vehículo de una racionalidad instrumental queenlaza sus objetivos a las tendencias de la globalización. Ante dicho estado de cosas, voces críticas alertan el desplazamiento de la filosofía a posiciones periféricas en el sistema educativo. Por lo anterior, es necesario repensar el papel de la filosofía en la educación. A este respecto, el objetivo del trabajo va dirigido a presentar las ideas centrales de una concepción del filosofar que reconsidera su posición en la escuela. En esta perspectiva, el filosofar se considera como un conjunto de acciones realizadas por docentes y estudiantes para intervenir en su realidad específica, lo que da lugar a la construcción de una mirada que pone bajo la duda aquello que mundanamente se considera inalterable. Se defiende la idea de que el filosofar en las escuelas inicia con un proceso de sensibilización que nos ayuda a des-aprender, con el fin de empezar a comprender el mundo de otras maneras. A todas luces, esta tarea resulta pertinente en el periodo de educación formativa.

Palabras clave: Filosofar, educación, experimental, humanizar, sensibilización.

\section{Abstract:}

The article discusses the role that the philosophy gets inside the curriculum, under the light of experimented changes of the educative system in the last decades. Currently, we have to recognize that school has modified its attention and perspective. It has gone from a knowledge provider and guidance of a humanistic formation to a manager of competencies and vehicle of instrumental rationality that links its objectives to the global trends. Considering such a state, critical voices warn about the shift of philosophy towards peripheral positions in the educative system. Consequently, it is necessary to think again the role of philosophy in education. By considering this, our work aims to present the central ideas of a conception of philosophizing that reconsiders its position inside the school. In this perspective, philosophizing is considered as a set of actions done for teachers and 
students to intervene in their specific reality, which allows the building of a look that questions whatever is considered mundanely unchangeable. It defends the idea that philosophizing in school begins with a process of sensitization that helps us to unlearn, in order to begin to understand the world in other ways. Finally, this task results relevant in a period of formative education.

Key-word: Philosopher, education, experimental, humanize, awareness.

\section{Vers une conception expérimentale de la philosophie à l'école}

\section{Résumé :}

Cet article traite le rôle qui prend la philosophie dans le curriculum après les changements qui l'ont arrivé pendant les derniers décennies. Actuellement, l'école est reconnue par la modification faite à son centre d'attention et à ses perspectives, car elle est passé d'être fournisseur de connaissances et guide d'une formation humanisteà gestionnaire de compétences et véhiculé d'une rationalité instrumentale qui lie ses buts avec les tendances de la globalisation. Par rapport à ces situations, des voix de critiques préviennent le déplacement de la philosophie à des positions périphériques dans le système éducatif. Comme conséquence, il faut repenser le rôle de la philosophie dans l'éducation. Face à cette situation-là, l'objectif du travail s'oriente à la présentation des idées centrales d'une conception de l'acte de philosopher qui prend en reconsidération sa place à l'école. Dans cette perspective, philosopher est vu comme un ensemble d'actions faites par des enseignants et des apprenants pour intervenir dans leur réalité spécifique, donnant comme résultat la construction d'une vision que met en doute cela qui en vain est considéré immutable. On défend l'idée qui affirme que la philosophie dans les écoles commence avec un processus de sensibilisation qui nous donne de l'aide pour désapprendre, avec l'intention de commencer à comprendre le monde d'autres manières, donc, cette idée devient pertinente dans les phases qui composent l'éducation formatrice.

Mots-clés : Philosophe, éducation, expérimental, humaniser, conscience. 


\section{Rumo a uma conceição experimental da filosofia na escola}

\section{Resumo:}

O artigo discute o papel que ocupa a filosofia no curriculum, à luz das mudanças experimentadas pelo sistema educativo nas ultimas décadas. $\mathrm{Na}$ atualidade, se reconhece que a escola tem mudado seu foco de atenção e perspectiva, pois passou de ser provedora de conhecimento e guia de uma formação humanistica a gestora de competências e veículo de uma racionalidade instrumental que enlaça seus objetivos à tendências da globalização. Ante esse estado de coisas, vozes criticas alertam o deslocamento da filosofia para proposições periféricas no sistema educativo. Por isso, é necessário repensar o papel da filosofia na educação. Neste aspecto, o objetivo do trabalho se encaminha a presentar a ideia central de uma conceição do filosofar que reconsidera sua posição na escola. Nesta perspectiva, o filosofar se considera como um conjunto de ações realizadas pelos professores e estudantes para intervir na sua realidade especifica, resultado da construção de uma mirada que coloca em dúvida aquilo que mundanamente se considera inalterável. Defina-se a ideia de que o filosofar nas escolas inicia com um processo de sensibilização que nos ajuda a des-aprender, com o fim de começar a compreender o mundo de outras maneiras. Assim, esta tarefa resulta necessária no período de educação formativa.

Palavras chave: filosofar, educação, experimental, humanizar, sensibilização.

\section{Introducción}

Este artículo surge de la pregunta ¿por qué necesita la escuela enseñar filosofía? Nos interesa plantear qué hay de particular en esta disciplina; particularmente, qué puede ofrecer en los niveles previos al ingreso a la universidad. El asunto no es nuevo, dado que ciertamente la filosofía ha sido cuestionada muchas veces, el juicio contra Sócrates es solo un ejemplo. Aquí, sin embargo, nos interesa ofrecer una respuesta al problema teniendo en cuenta tres aspectos: primero, un diagnóstico acerca del lugar que ocupa la filosofía en la escuela; segundo, establecer los principales retos que enfrenta la filosofía en la escena educativa; $y$, tercero, delinear los elementos filosófico-pedagógicos de una concepción del filosofar que replantee su rol actual. 


\section{El juicio contra la filosofia}

Como resultado de los cambios que están experimentando actualmente nuestras sociedades, hay un cuestionamiento a la enseñanza de la filosofía en las escuelas. La idea subyacente proviene del siguiente supuesto: la filosofía no ofrece ningún conocimiento específico que dote de alguna habilidad para la vida a los jóvenes que estudian (Vargas, 2011). Nuestras sociedades enfrentan maneras de comunicarse, interactuar, consumir y estudiar que antes eran desconocidas. Esto, a todas luces, exige repensar las maneras de enseñar y aprender. Ante ese esfuerzo, los enfoques más pragmáticos del cambio educativo centran su atención en los aspectos prácticos del aprendizaje, lo que termina por desplazar a las disciplinas teóricas a una posición periférica (Raschke, 2003; Pacheco-Méndez, 2015; Rius, 2012; Vargas, 2011).

Los procesos de globalización ejercen influencia en las instituciones educativas, de tal modo que se convirtieron en el factor principal de orientación de los fines de la escuela. El tiempo de la enseñanza y los espacios para generar el aprendizaje se encuentran en transformación (Oblin, 2014). La escuela se ha convertido en un vehículo más de la racionalidad instrumental y ha terminado por trasladar sus fines a los procesos de la globalización, con lo que ha borrado sus diferencias específicas y se ha asimilado como una mercancía más sometida al proceso de oferta y demanda (Samper, 2002). Distintos paises de lberoamérica ${ }^{22}$ experimentan la transición de un modelo tradicional de enseñanza y gestión educativa, con tintes humanísticos e ilustrados, a otro de administración del conocimiento y orientación a la competitividad. Bajo este contexto, la filosofía por su condición de disciplina "teórica" ha sido relegada.

2 La reforma educativa española (LOMCE) redujo las asignaturas filosóficas de forma sustancial en los programas de estudio; ahora se enseñan en secundaria y bachillerato tres asignaturas relacionadas: Filosofía, Valores Éticos e Historia de la Filosofía. Solo la primera será obligatoria en $1^{\circ}$ de bachillerato. En México, la Reforma Educativa (RIEMS) convirtió las asignaturas filosóficas para bachillerato en Ética y valores I y II, lo que deja para el último semestre del bachillerato un solo curso de Filosofía. 


\section{La filosofía en el contexto sistémico}

Responder a la pregunta de por qué necesitamos enseñar filosofia hoy exige retomar tres cuestiones: 1) cuál es la naturaleza de la filosofía; 2) cuál es su sentido en la vida de las personas; y 3) para qué integrarla al sistema educativo. Respecto de la primera cuestión, suele decirse que la filosofía carece de una definición u objeto particular. A pesar de que Fichte insistía en que la filosofía era la ciencia de las ciencias, en la actualidad resulta discutible tal caracterización (Wilson, 1998).

A lo anterior, podemos sumar el impacto de la filosofía en cualquier dominio de la vida. Para muchos, la única responsabilidad que le compete al filósofo es la de ofrecer argumentos (Baggini, 2011), pero estos parecen tener un efecto mínimo en el mundo instrumental (Oblin, 2014; Pacheco-Méndez, 2015). Así, la filosofía parece proyectar limitadas expectativas en la educación. Incluso la reflexión acerca de la condición humana se ha vuelto el objetivo de otras disciplinas; por ejemplo, la robótica, la economía o la psicología.

La escuela y la filosofía padecen el embate de la racionalidad sistémica, la cual se guia por dos principios: diferenciación y especialización (Luhmann, 1999). Entiéndase sistémico como un tipo de orden guiado por objetos de un sistema. "[E]lementos que se hallan entre si en relación funcional tal que se produce una interdependencia de acuerdo con un conjunto de reglas" (Ferrater, 1979, p. 3068). De acuerdo con Luhmann (1999), las condiciones establecidas por los enfoques educativos contemporáneos revisten tendencias sistémicas, porcuanto su orientación va dirigida hacia la eficiencia práctica; por ello, tienden a cercenar la parte fundamental de lo humano (Kohan, 2014; Hoyos, 2012).

Niklas Luhmann nos advirtió en sus estudios que los principios del orden sistémico causarian en las instituciones y en las disciplinas tendencias de homogeneización, autoatribución y diferenciación (1996, p. 43). Lo anterior, ahora entendemos, significa que tanto la escuela como la filosofía mantienen su presencia en el sistema social al costo de adoptar la forma de las estructuras del sistema, al autoreproducirlo permanentemente. El punto aquí es que la enseñanza de la filosofía está presente en el sistema solo a condición de su reproducción bajo el orden del sistema, ya que 
de lo contrario desaparecería. El asunto nodal ahora es el siguiente: qué significa en este contexto que se reproduzca. Claramente, lo que se reproducen son sus contenidos declarativos, sus procedimientos, mas no hay cabida para el ejercicio de pensar sobre el pensamiento. Dentro del enfoque sistémico, llevar a los estudiantes a pensar de manera auténtica es, como afirma Emilio Martínez Navarro (2012), un "acto decorativo".

Ahora bien, lo anterior también nos presenta los ángulos débiles del enfoque sistémico. Efectivamente, la escuela debe desarrollar habilidades para enfrentar nuestros problemas inmediatos, no solo para pensarlos. Sin embargo, para determinar cómo resolver un problema, es insuficiente decir que basta con fomentar destrezas, también es indispensable señalar cómo y de qué manera adquiere pertinencia un problema. Consideremos lo siguiente: aunque es cierto que la filosofía no tiene un objeto específico, realiza tareas para las cuales otras disciplinasestán incapacitadas. Pensemos en el siguiente ejemplo: para la economía doméstica de una persona es importante saber distribuir sus gastos, con el fin de tener un balance entre lo que se tiene y se gasta. Se puede argumentar que los conocimientos de administración y economía, tal como se hace ahora en las escuelas de educación secundaria bastan; empero, el manejo del dinero siempre va acompañado de adjetivos: queremos usar bien el dinero, optimizar nuestros ingresos, etcétera.

Para cumplir con lo anterior, se necesita determinar en qué consiste usar bien el dinero, evaluar ventajas y desventajas, analizar tipos de beneficios $y$, si somos un poco más exigentes, podriamos considerar una revisión de nuestros supuestos: a qué y cuándo atribuimos adjetivos como "mejor" u "óptimo". Una revisión detenida de nuestras creencias y conceptos, podría arrojar información relevante acerca de cómo entendemos asuntos que en lo convencional no tienen nada de problemático. Los procesos de emisión de juicio, evaluación y contrastación de nuestras creencias requieren algo más que un saber práctico.

\section{Sentido de la filosofía y el filosofar}

Hemos presentado algunas dificultades que enfrentan la educación y la filosofía dentro dela perspectiva sistémica, ahora necesitamos explorar la noción de filosofía. 
En este punto, argumentaré que la filosofía en la escuela es más que un conjunto de conocimientos, métodos y teorías, sino que es una manera de interactuar con el mundo, lo cual abre campos de comprensión de la realidad distintos a los ofrecidos por las disciplinas científicas y sociales.

Considérese la siguiente formulación de Bertrand Russell:

Todo conocimiento definido pertenece a la ciencia -asi lo afirmaria yo-, y todo dogma, en cuanto sobrepasa el conocimiento determinado, pertenece a la teología. Pero entre la teología y la ciencia hay una Tierra de nadie, expuesta a los ataques de ambos campos: esa Tierra de nadie es la filosofía (2009, p. 33).

La caracterización de Russell ofrece una dimensión del saber humano ubicado entre lo definido y lo incuestionable. Eso es, la filosofía no tiene un objeto específico porque no lo necesita; su ocupación está en aquello que los demás saberes no tienen en cuenta. La filosofía, por su condición, crea una dimensión de interrogación propia, llena de espacios para acercarse al mundo. Bajo la anterior consideración, si la filosofia se encuentra entre lo definido y lo incuestionable, su campo de trabajo está abierto permanentemente; es, a decir de Russell, una "Tierra de Nadie". De lo anterior, podemos colegir la débil justificación en el reproche contra la filosofía. El modelo educativo actual, por su condición sistémica, es incapaz de concebir disciplinas sin objeto. A continuación, argumentaremos más abundantemente sobre este punto.

Indaguemos las maneras de entender la actividad filosófica: primero, por sus significados; luego, por sus fines. Para empezar, existe un sentido laxo para hablar de la filosofía, dado que se le atribuye a cualquier persona que razone sobre asuntos generales como el significado de la vida, la educación o la justicia; en contraparte, hay un segundo sentido que relaciona al filósofo con un profesional formado en ciertos estudios académicos (Phillips y Siegel, 2015).

La primera caracterización no requiere análisis, aceptarlo es reconocer simplemente que la filosofía no necesita institucionalizarse, reflexionar sobre asuntos importantes de la vida no es exclusivo de algunos profesionales; además, quienes se preocupan 
de tales asuntos ni siquiera necesitan estar informados de disciplinas y métodos. La segunda, en cambio, nos pone frente a la exigencia de justificar por qué un filósofo debeser un profesional certificado bajo criterios de una comunidad institucionalizada. Una razón para admitir la filosofía como disciplina dice que los filósofos profesionales, a diferencia de los no profesionales, se toman la tarea de "regular el flujo de ideas" (Baggini y Stangroom, 2011, p.11) generado por las actividades de pensamiento y atendiendo a los criterios de la argumentación racional. En este sentido, el filósofo si requiere una formación específica para saber exponer sus ideas, respaldarlas con evidencias y construir argumentos.

La segunda manera de abordar el problema de la enseñanza de la filosofía consiste en plantearnos cuáles son sus fines en la educación. Para lo anterior, se han ofrecido dos respuestas. La primera podría presentar la siguiente formulación: la escuela debe dedicarse a ofrecer los elementos necesarios para acercar a las personas a un ideal que favorezca su florecimiento; el filósofo es entonces un educador que guía hacia un ideal de perfección humana. La segunda respuesta dice que la escuela no tiene que orientar a nadie a ningún ideal, basta que el profesor dote a sus estudiantes de herramientas para alcanzar una vida autónoma.

Una objeción a la idea de que el profesor-filósofo guia hacia la buena vida es que no le compete a ningún funcionario educador intervenir en la definición de los ideales de vida de las otras personas. Otra objeción sostiene que modelar el comportamiento de los estudiantes no se logra atendiendo a contenidos declarativos, ya que la escuela y el docente no deben exigir que sus estudiantes se adscriban a una concepción del bien determinada. La objeción a la segunda formulación es que las instituciones educativas no tienen entre sus principios normativos la obligación de preparar para la virtud o la autonomía. Más bien, su tarea es la de fomentar en sus estudiantes la consciencia de que son sujetos de derecho (Lorandi, 2009; Ramírez, 2006).

Volvamos nuevamente a nuestra caracterización inicial de la filosofía. Quien habita la filosofía enfrenta la educación de una manera diferente a como lo hace el Estado, la moral, la ciencia o la religión. El espacio de observación del filósofo es un ángulo no reductible a lo mundano ni a las descripciones normativas. Pero ¿qué significa eso? Sencillo, que su perspectiva remite a la externalidad. La filosofía se convierte en un 
mirador inédito del mundo. Si aceptamos que la filosofía nos coloca en un lugar que no está ocupado por una instancia concreta, las acciones filosóficas son educativas porque construyen un camino, con sus reglas y su orden; además, requieren de la guia de un experto para esclarecer el camino.

La tarea educativa de la filosofia tiene interés en orientar al estudiante a encontrar su camino. En gran medida, la construcción de ese camino es el objetivo del filósofo en la enseñanza; ser filósofo es ser un acompañante en la construcción de la vía de ser de cada joven. Esa tarea, a diferencia de la idea de vida buena, de la vida competente, de la vida orientada por las definiciones estrictas o los dogmas, tiene como fin al propio estudiante. En este sentido, el acto educativo del filósofo es formativo. Educar consiste en ayudar a los más jóvenes a aprender a ser ellos mismos, como lo pensara Hanna Arendt. El profesor, en su rol de filósofo, ayuda a cada estudiante a "convertirse en un ser humano" (Arendt, 2016, p. 292). Pero es importante indicar que la enseñanza del filósofo no es inocua, ya que puede ser alienante; sinembargo, también puede producir un efecto liberador sobre la dominación arbitraria que el mundo adulto impone sobre el mundo joven (Alba-Meraz, 2014, 2008; Conde-Flores, Garcia-Cabrero y Alba-Meraz, 2017).

\section{Estructura de la enseñanza de la filosofía}

Planteadas las razones que justifican la presencia de la filosofía en el currículum, en esta sección tenemos el objetivo de presentar nuestra concepción de la enseñanza de la filosofía. Primero que todo, recuperaremos nuestra formulación del acto de filosofar; luego, ofreceremos una descripción de cómo se entiende el filosofar a través de la interacción del aprendiz en el proceso educativo; finalmente, señalaremos cómo se reflejan esas acciones en la formación del estudiante.

Desde un punto de vista didáctico y metodológico, nos parece importante distanciarnos, primero, de las formas que evitaremos al enseñar filosofía. A estas las llamaremos la manera temática y la de enfoque figurativo (Nobis, 1999). Cuando hablamos de enseñanza "temática" hacemos referencia al estudio de los "tópicos" o "problemas" que se encuentran en los programas centrados en contenidos: métodos, tipos de argumentos, limites de la formalización, los universales, crítica 
de la razón, etcétera. Por enseñanza "figurativa" queremos indicar esa orientación dirigida a las respuestas ofrecidas por las figuras destacadas de la historia de la filosofía; por ejemplo, Aristóteles, San Agustín o Hegel.

Estas maneras de concebir la enseñanza presentan la actividad filosófica descontextualizada y ofrecen una mirada fragmentada del corpus del filósofo estudiado como conjunto de presentaciones de tesis, respuestas o soluciones a problemas sin considerar; por ejemplo, sus herencias con las tradiciones previas. De acuerdo con Johannes Rohbeck (2014), todo esto destruye gran parte de su comprensión. Los estudiantes durante su educación formativa, e incluso durante los primeros años en la universidad, carecen también de una iniciación a la experiencia de consultar fuentes primarias, lo que reduce su estudio a interpretaciones y comentaristas. Asimismo, desconocen el uso de los métodos filosóficos de interpretación de los textos. Ambas cosas exigen una formación en habilidades especificas que otorgan una comprensión puntual y técnica de las cuestiones (AlbaMeraz, 2019; Applis y Tiedemann, 2014; Rohbeck, 2014).

Otra crítica a los modos temático y figurativo está centrada en su pretensión de transmisión de conceptos abstractos o de habilidades de aplicación. La expresión general de esta crítica consiste en aceptar el supuesto de que los estudiantes atrapan conceptos abstractos con la mente, como resultado de la transmisión de estos a partir de definiciones y que luego se comentan y se aplican en ensayos. La anterior es, de acuerdo con Jay Lemke (1994), una visión encantada del aprendizaje. Concordamos en que los estudiantes necesitan aprender teorias y conceptos, el punto, sin embargo, es cómo lograrlo, cómo se produce una idea, cómo se logra abrir una nueva ventana al mundo. Tal asunto difícilmente se conseguirá a través de una clase convencional en la que se ofrecen definiciones y conceptos (Kirshner y Whitson, 1997; Bourdieu, 1990). En cambio, actualmente se explora la educación filosófica a partir del modelamiento del uso de los métodos, es decir, llevando al estudiante a experimentar la actividad filosófica (Rohbeck, 2014; Cholbi, 2007).

Nuestra concepción de la enseñanza de la filosofía pone el acento en el filosofar, pues considera a los estudiantes aprendices del oficio de filosofar y al docente un filósofo. Esta idea puede formularse como una concepción experimental del 
filosofar. Aquí el aprendizaje consiste en un conjunto de acciones de intervención pedagógica, en donde la reflexión se produce como resultado de sensibilizar para aprender a escuchar, no responder anticipadamente, ejercer la critica, aclarar ideas y conceptos, argumentar correctamente, tener confianza para preguntar y buscar soluciones no convencionales, además de dedicarse a preparar textos en distintos formatos.

Nuestra concepción filosófico-pedagógica tiene sus antecedentes en la propuesta del filósofo alemán Johannes Rohbeck (2007, 2008 y 2014), quien considera la enseñanza de lafilosofía como la acción de modelar el uso de los métodos filosóficos. El principal aporte didáctico de este enfoque consiste en proponer un sistema de ayudas -de parte del docente al estudiante-para crear destrezas en el manejo de los métodos. De acuerdo con Rohbeck, la práctica filosófica en la escuela consiste en la coordinación de las formas (direcciones) de pensary el uso de los métodos filosóficos de enseñanza (2007, pp. 100-101). En otras palabras, se trata de la transformación de las maneras de pensar en estructuras sistematizadas que pueden aprender los estudiantes y aplicar. En eso consiste el proceso de modelado filosófico. De nuestra parte, consideramos que existen limitaciones en el argumento de Rohbeck, ya que no basta con mostrar el procedimiento para darle sentido a la práctica filosófica; de hecho, la pretensión de que son las habilidades procedimentales las que conducen a la comprensión sigue manteniendo la raíz del cuento de hadas.

Mostrar y modelar el uso del método es necesario pero no suficiente; ese procedimiento se centra principalmente en las facultades cognitivas del aprendiz y, por supuesto, del profesor. Además, lleva a la base una imagen racionalista del aprendizaje y del filosofar. A nuestro juicio, los actos de filosofar no son solamente acciones de racionalización de procedimientos, sino que son en gran parte acciones motivadas que tienen como base las emociones. Por lo mismo, el principal aspecto que mueve la necesidad de experimentar el filosofar es emocional.

Las personas antes de empezar a filosofar o usar un método filosófico requieren primero estar motivadas o encontrarse en disposición para ello; deben estar dispuestas a la experiencia de pensar. En este sentido, filosofar es una acción que viene a ser el resultado de un estado intencional-emocional. Por ello, para que una 
persona sienta la necesidad de pensar como un filósofo, debe sentirse primero como un filósofo, sentir las inquietudes de querer preguntar. Cuando esto sucede, empieza el filosofar (Peña, 1982). Así pues, la filosofía es una actividad que transcurre en un proceso de reconstrucción emocional-racional (Lemke, 1997).

Nuestra perspectiva considera entonces un paso previo para la coordinación entre direcciones de pensamiento y métodos. Dicho paso es el espacio para sentir lo que se aprende, lo cual es una práctica constitutiva del ser humano (Rohbeck, 2007; Kopfwerk, 2005; Lemke, 1994; Peña, 1982; Barth y Krabbe, 1982). De esta manera, el experimentar la filosofía tiene un proceso de sensibilización previo a la investigación de los problemas. El acercamiento a los problemas inicia, como sabemos, con el asombro; el surgimiento de las preguntas es posterior. Las preguntas son el resultado de experiencias de asombro ante lo planteado en el mundo, como se dijo, en relación con asuntos del entendimiento común (Lee, 2018; Kopfwerk, 2005; Barth y Krabbe, 1982).

En cuanto a las preguntas, estas emergen cuando son asuntos relacionados con lo común, pues al estudiante le interesa entender su condición en el mundo. Tales preguntas, si el estudiante está emocionalmente dispuesto, pueden ir a dimensiones más profundas. Cuando el estudiante ha llegado a sentirse interesado por lo que lo rodea, puede ampliar el espectro de sus preocupaciones.

Nuestra metodología de trabajo ha planteado el valor normativo que tiene la fase de sensibilización en la enseñanza de la filosofía, luego vendrán los aspectos procedimentales (como aprender a formular preguntas). Todo ello debe tener lugar antes de pasar a los conceptos abstractos. Para sintetizar: el propósito consiste en explorar las inquietudes de los estudiantes, al estimular sus emociones. Ahora bien, no nos interesa adentrarnos en los procesos psicológicos ni en los estilos de aprendizaje de los estudiantes. Lo que en realidad nos interesa es sensibilizar la experiencia con lo mundano, para luego iniciar el proceso de formulación de las preguntas. Lo obvio, necesario o poco apreciable pueden convertirse en espacios de exploración de la experiencia humana, lo que puede llevarnos a distintos niveles de preocupación, asombro y finalmente cuestionamiento. Despertar interés por nuestras certidumbres e inseguridades -para ir poco a poco encaminados a la 
elaboración de preguntas- es algo que Sócrates practicaba permanentemente (Kopfwerk, 2005). Esa acción es una forma de habitar la tierra de nadie de manera distinta a como lo hacen las otras disciplinas.

Al considerar cuidadosamente el tema del filosofar como un des-aprender lo conocido para aprender a construir un camino propio, sin recurrir permanentemente a las definiciones convencionales, importa el profesor y su papel de diseñador de las experiencias de sensibilización y pensamiento. La razón estriba en que quien ayuda a modelar sobre lo desaprendido debe ser alguien experimentado en el proceso, tener el manejo de la experiencia de sensibilizar, dominar los métodos filosóficos y las teorias.

Las acciones y la distribución del tiempo destinado para sensibilizar o desarrollar habilidades y para preguntar o apropiarse de los conceptos son importantes, pero difieren sustancialmente. De manera general, elaprendizaje de conceptos abstractos domina el uso del tiempo. Sabemos, sin embargo, que es necesario establecer dimensiones temporales para el aprendizaje: la temporalidad individual, la cual nos informa acerca del contexto presente, a través de las experiencias cotidianas, y la temporalidad inherente al ejercicio del pensar filosófico que tiene su campo en el futuro (Zimbardo y Boyd, 2008). El conflicto con el tiempo es que las actividades de sensibilización y construcción de capacidades para sentir y las de los actos de pensar no siempre se sincronizan.

La mayoría de quienes están familiarizados con la filosofía saben que "alumbrar una idea" es el resultado de un camino largo y que va precedido de múltiples momentos, por lo cual el tiempo dedicado a crear una disposición es largo. La amplitud del tiempo para conseguir una idea va precedida de aprender a sentir la duda, iniciar un camino para la reflexión, aprender a escuchar con atención, evitar juicios apresurados, revisar nuestras perspectivas, intercambiar diálogos e iterativamente volver al inicio. Lograr esas acciones puede permitir continuar con la elaboración de escritos filosóficos, como ensayos o disertaciones, para los cuales se necesita dedicar un tiempo amplio para redactar, aclarar conceptos o incorporar ideas a una explicación, formar una hipótesis o construir un argumento. Lo filosóficoteórico toma forma después de muchas acciones emocionales e intelectuales. 
Dado que los programas académicos están orientados a resultados, es decir, al cumplimiento de un objetivo predeterminado, dicho espectro temporal entra en conflicto, como afirman Zimbardo y Boyd (2008), con la temporalidad del aprendiz, lo cual regularmente se convierte en frustración, enojo o apatía.

La relación entre la construcción de experiencias y la capacidad de asombro para pensar transcurre en un tiempo que se cruza con la temporalidad de otro binomio (transmisión-transferencia) que es el que suele definir las acciones didácticas; por ello, es importante realzar el sentido que tiene el tiempo en la Tierra de nadie. Si el filosofary la filosofía son maneras en las que la experiencia y el pensamiento se ponen en contacto para darle un sentido al mundo, estamos señalando que no hay recetas de distribución del tiempo, así como no tienen un privilegio los procesos cognitivos en las formas de aprender, dado que hacerlo definiria ya la manera de filosofar. La manera de establecer un vínculo significativo con la filosofía ocurre cuando se lleva a cabo la conjunción entre una perspectiva emocional, una dimensión temporal adecuada, un campo de preocupaciones, creencias, ideas y un método. El resultado de todo esto es un cambio en las maneras de aprender.

La propuesta delineada del filosofar es viable y puede recrearse como un quehacer cotidiano, pero es necesario decir que también requiere de la disposición del docente. Un profesor debe presentar su concepción como ejemplo de ejercicio filosófico. Sentir la toma de postura es lo que le da sentido a las orientaciones de pensamiento y ello en conexión con un método genera una práctica filosófica (Rohbeck, 2008, pp. 12-14). En la práctica, una determinada concepción no cancela otras formas de hacer filosofía; el profesor tampoco debe ocultar ni mostrarse como juez inmunizado contra las emociones. En este sentido, la filosofía y el filosofar son dos caras de una misma moneda, en donde tenemos experiencias emocionales de la reflexión, la crítica, la dilucidación o la argumentación.

Ya en el plano de las acciones posteriores a la sensibilización, la enseñanza de la filosofía requiere establecer qué debe suceder: pensar criticamente, plantearse problemas, estructurar pacientemente un argumento, pero sobre todo saber cuándo poner en ejercicio la duda (Peña, 2009, p. 2). Enseñar a filosofar es experimentar la duda y disfrutar del ejercicio de pensar. Enseñar deliberadamente a los estudiantes 
cómo formular preguntas es una auténtica tarea filosófica y, en un nivelmás profundo, este ejercicio permanente lo convierte en una persona para quien el cuestionamiento es un hábito. Finalmente, las razones aqui vertidas son la respuesta a la pregunta de por qué la filosofia necesita continuar entre nosotros en las escuelas.

\section{Conclusiones}

En este trabajo, hemos descrito cómo las condiciones de la crisis actual afectan a la educación en general y a la filosofía en particular. Las causas de esta crisis están en los procesos y lógicas de la globalización neoliberal. En este contexto, nuestro primer objetivo fue presentar la manera en que las reformas del sector educativo cuestionan la pertinencia de la filosofía en el currículum, dado su carácter teórico y poco práctico. Sin embargo, como hemos argumentado, los intereses de las personas carecen de sentido, sino agregamos algunas acciones que la filosofía fomenta, verbigracia: hacer preguntas y revisar nuestras creencias acerca del mundo presente. El reto que tenemos enfrente, quienes nos dedicamos a la filosofía, pero en especial a la filosofía de la educación, es re-significar el rol de nuestra disciplina y mostrar por qué su presencia en los programas educativos es necesaria.

Al encauzar el análisis a la caracterización de la filosofía en la escuela, hemos tratado de mostrar que, si bien no contamos con una definición estándar, su condición de espacio abierto, distinto al de la ciencia o la religión, nos pone frente a un horizonte de exploración y construcción de sentido de nuestra existencia. Este proceso puede entenderse como la constitución de nuestra humanidad, hecho insustituible, para lo cual no contamos con otra disciplina afín. Aunque se ha señalado que la filosofía puede encontrarse fuera de la escuela, también es un hecho que la formación en la disciplina nos permite desarrollar otras habilidades que complementan el asombro cotidiano; por ejemplo: aprender a escuchar, atender a las contradicciones, tener una metodología para preguntar o definir una guia para ofrecer soluciones.

La rehabilitación de la filosofía en la escuela genera una deconstrucción de la realidad del estudiante y del docente, de manera tal que nos permite conciliar y articular los fragmentos del mundo circundante, algo que nos encauza a re-significar nuestro mundo y nuestra existencia. Por último, el planteamiento didáctico, formulado como 
un marco de intervención filosófica en el aula, muestra que la reestructuración de la enseñanza necesita considerar, además del enfoque de intervención basado en la experimentación de la filosofía, la restauración de aspectos como la temporalidad. La definición del docente en favor del tiempo del filosofar es una condición para hacer posible la concepción experimental del filosofar, la cual debe encontrar un equilibrio con la temporalidad institucional, con la estructura sistémica de las asignaturas y con las reticencias de los estudiantes a pensar como filósofos. Pese a las dificultades que entraña lo anterior, consideramos que el ejercicio filosófico es posible en las aulas, pero, sobre todo, estamos convencidos de que tener filosofía en las escuelas genera una oportunidad digna para construir una comunidad de seres humanos.

\section{Referencias}

Alba-Meraz, A. (2008). La cultura política en la escuela. Un acercamiento a los conflictos entre moral, cognición y lo político. G. Gutiérrez Castañeda (Coord.), Cuatro eslabones para pensar la cultura política (pp. 49-80). Ciudad de México: Universidad Nacional Autónoma de México.

Alba-Meraz, A. (2014). Conflicto, autoridad y argumentación. Elementos para pensar los caminos para la paz en el aula. Revista Sinéctica, (42), pp. 59-74.

Alba-Meraz, A. (2017). El aprendizaje de la filosofía y la cultura del texto escrito. R. Picos Bovio (Coord.), Didáctica de la Filosofia. Prácticas, retos y expectativas (pp. 89108). Ciudad de México: Editorial Itaca.

Alba-Meraz, A. (2019). Aprendizaje y evaluación de la lógica. G. Vadillo (Ed.), Evaluación de los Aprendizajes en el Bachillerato (pp. 3-10). Ciudad de México: Universidad Nacional Autónoma de México.

Applis, S., y Tiedemann, M. (2014). Experimentelle Philosophiedidaktik. Lernen von den Nachbarwissenschaften. J. Rohbeck (Ed.), Experimentelle Philosophie und Philosophiedidaktik (pp. 70-87). Dresde: Thelem. 
Arendt, H. (2016). Entre el pasado y el futuro. Ocho ejercicios sobre la reflexión política. (Trad. A. Poljak). Barcelona: Ediciones Península.

Baggini, J., y Stangroom, J. (2011). Lo que piensan los filósofos. (Trad. P. Hermida Lazcano). Barcelona: Paidós.

Barth, E. M., y Krabbe, E. C. (1982). From Axioma to Dialogue. Berlin: Walter de Gruyter.

Bourdieu, P. (1990). The logic of practice. Stanford: Stanford University Press.

Cholbi, M. (2007). Intentional Learning as a Model for Philosophical Pedagogy. Teaching Philosophy, 1(30), pp. 35-58.

Conde-Flores, S., Garcia-Cabrero, B., y Alba-Meraz, A. (2017). Civic and Ethical Education in México: From Classic Civics to the Development of Civic and Citizenship Competencies. B. García-Cabrero, A. Sandoval-Hernández, E. Treviño-Villarreal, S. Diazgranados Ferráns, M. G. Pérez-Martínez (Eds.), Civics and Citizenship. Theorical models and Experiences in Latin America (pp. 41-66). Rotterdam: Sense Publishers.

Ferrater, J. (1979). Diccionario de Filosofía. Madrid: Alianza Editorial.

Hoyos, G. (Ed.). (2012). Filosofía de la Educación (Enciclopedia Iberoamericana de Filosofia). Madrid: Editorial Trotta.

Kirshner, D., y Whitson, J. (Eds.). (1997). Situated Cognition. Mahwah, New Jersey, London: LEA Publishers.

Kohan, W. (2007). Sobre las antinomias de enseñar filosofia. Cuestiones de Filosofia, (9), pp. 143-160.

Kopfwerk, B. (2005). The Methodology of Socratic Dialogue. J. P. Brune y D. Krohn (Eds.), Socratic Dialogue and Ethics (pp. 88-111). Münster: LIT Verlag.

Lemke, J. (1994). Semiotics and the Deconstruction of Conceptual Learning. Journal 
of Accelerative Learning and Teaching, 19(1), pp. 67-110.

Lemke, J. (1997). Cognition, Context, and Learning: A Social Semiotic Perspective. D. Kirshner y J. Whitson (Eds.), Situated Cognition (pp. 37-55). Mahwah, New Jersey, London: LEA Publishers.

Lorandi, M. (2009). Viviendo y promoviendo nuestros derechos. Una mirada hacia la infancia y la adolescencia en México (pp. 121-171). Ciudad de México: Debate.

Luhmann, N. (1999). Teoría de la sociedad y pedagogía. (Trad. C. Fortea). Barcelona: Paidós.

Martínez Navarro, E. (2012). La Filosofía en la enseñanza secundaria en España: balance y perspectivas. Padres y Maestros, (343), pp. 40-43.

Nobis, N. (1999). Cultivating Philosophical Skills and Virtues in Philosophy of Education. Revisado el 20 de mayo de 2018, en http://www.nathannobis.com/ papers/Cultivating\%20Philosophical\%20Skills\%20an d\%20Virtues\%20in\%20 Philosopy\%20of\%20Education.pdf.

Oblin, N. (2014). La escuela ante la racionalidad contemporánea. ¿Dónde queda el pensamiento? Constelaciones. Revista de Teoria Crítica, (6), pp. 51-83.

Pacheco-Méndez, T. (2015). Desafíos del pensar social y humanistico frente a las tecnologias de la información y la comunicación. Ludus Vitalis, 23(43), pp. 289-300.

Peña, L. (2009). Consideraciones sobre el método de la enseñanza universitaria de la filosofía. Boletín de la APPUCE. Revisado el 5 de mayo de 2018, en http://digital.csic. es/bitstream/10261/15383/1/ensefilo.pdf.

Phillips, D. C., y Siegel, H. (2015). Philosophy of Education. The Stanford Encyclopedia of Philosophy. Revisado el 10 de mayo de 2018, en https://plato.stanford.edu/ archives/win2015/entries/education-philosophy/. 
Ramírez, G. (2006). Avances y retos de la Educación en Derechos Humanos en el siglo XXI. De América Latina a México. J. Gutiérrez Contreras (Coord.), Educación en Derechos Humanos (pp. 179-206). Ciudad de México: Secretaría de Relaciones Exteriores.

Raschke, C. A. (2003). The Digital Revolution and the Coming of the Postmodern University. London-New York: Routledge \& Falmer.

Rius, M. (2012). Las humanidades en la era 2.0. Periódico La Vanguardia 14 de octubre 2012. Revisado el 14 de febrero de 2014, en http://www.lavanguardia.com/estilosde- vida/20111014/54229795673/las-humanidades-en-la-era-2-o.html [14 de febrero 2014]

Rohbeck, J. (2007). Transformación didáctica: direcciones de pensamiento de la filosofía y métodos de enseñanza. Diálogo Filosófico, (67), pp. 97-110.

Rohbeck, J. (2008). Didaktik der Philosophie und Ethik. Dresde: Thelem.

Rohbeck, J. (2014). Proto-Ethik in didaktischer Absicht. J. Rohbeck (Ed.), Experimentelle Philosophie und Philosophiedidaktik (pp. 114-125). Dresde: Thelem.

Russell, B. (2009). Historia de la Filosofía. (Trad. J. Gómez de la Serna y A. Dorta). Madrid: Espasa Calpe.

Samper, E. (2002). Educación y globalización. Educación y globalización: los desafios paraAmérica Latina (pp. 43-49). Santiago de Chile: OEI, CEPAL, Corporación Escenarios.

Vargas, G. (Comp.). (2011). La situación de la Filosofía en la Educación Media Superior. México: Editorial Torres Asociados/Red Internacional de Hermenéutica Educativa.

Wilson, E. (1998). Consilience: The Unity of Knowledge. London: Little, Brown and Co.

Zimbardo, P., y Boyd, J. (2008). The Time Paradox: The New Psychology of Time that Will Change your Life. New York: Free Press. 